\title{
ON THE COMMUTATIVITY OF RESTRICTED LIE ALGEBRA
}

\author{
BYOUNG-SONG CHWE
}

The purpose of this note is to prove the following.

THEOREM. A finite-dimensional restricted Lie algebra $L$ over an algebrically closed field $F$ with characteristic $p \neq 0$ is abelian whenever the p-map has no nontrivial zero.

Proof. Let $\phi$ denote the $p$-map in $L$. For $x \in L$, let $V_{x}$ be the subspace of $L$ that is spanned by $x, \phi(x), \phi^{2}(x), \cdots$. Clearly, $V_{\phi(x)} \subset V_{x}$. Also $V_{x}$ has a basis consisting of elements of the form $\phi^{k}(x)$. The images under $\phi$ of these basis elements are linearly independent elements of $V_{\phi(x)}$, for if $\sum_{k} a_{k} \phi^{k+1}(x)=0$, then

$$
\phi\left(\sum_{k}\left(a_{k}\right)^{1 / p} \phi^{k}(x)\right)=0,
$$

and so

$$
\sum_{k}\left(a_{k}\right)^{1 / p} \phi^{k}(x)=0 .
$$

Hence the dimension of $V_{\phi(x)}$ is at least as large as the dimension of $V_{x}$, whence $V_{\phi(x)}=V_{x}$. It follows that, for every positive integer $k$, $V_{x}=V_{\phi^{k}(x)}$. In particular, $x \in V_{\phi^{k}(x)}$. Let $D_{x}$ denote the inner derivation of $L$ that is effected by $x$. Then it follows immediately from our result that, for every $y \in L$ and every positive integer $n, D_{x}^{n}(y)=0$ implies $[x, y]=0$.

Now suppose that $[x, y] \neq 0$. Let $W$ be the subspace of $L$ that is spanned by $y, D_{x}(y), D_{x}^{2}(y), \cdots$. Since $D_{x}^{n}(y) \neq 0$ for any $n, D_{x}$ is not nilpotent on $W$. Hence there is a nonzero subspace $W_{1}$ of $W$ on which $D_{x}$ induces a linear automorphism. If we take $W_{1}$ of minimal dimension then it is irreducible and hence, by Schur's Lemma, 1-dimensional. Thus there is a nonzero element $z$ in $W$ such that $D_{x}(z)$ $=a z$, where $a$ is a nonzero element of $F$. This may be written $D_{z}(x)$ $=-a z$ and gives $D_{z}^{2}(x)=0$, whence $D_{z}(x)=0$, which contradicts $a z$ $=0$. Thus $[x, y]=0$, and we have shown that $L$ is abelian.

University of Alberta, Edmonton, Alberta, Canada

Received by the editors January 2, 1964. 